\title{
Cardiac Function, Substrate Utilization, and Myocardial Energy Metabolism Studied with 31-P NMR Spectroscopy during Acute Hypoglycemia and Hyperketonemia
}

\author{
JOHANNES BREUER,' KYUNG J. CHUNG, ERKKI PESONEN, RICHARD H. HAAS, \\ BRIAN D. GUTH, DAVID J. SAHN, AND JOHN R. HESSELINK \\ Departments of Pediatrics and Radiology, University of California at San Diego, School of Medicine, \\ La Jolla. California 92093
}

\begin{abstract}
Whether severe hypoglycemia alone or in combination with hyperketonemia might cause deterioration of cardiac function has been controversial. Therefore, the influence of acute hypoglycemia (mean $33 \mathrm{mg} / \mathrm{dL}$ ) with and without hyperketonemia (mean 1.3 and $3.3 \mathrm{mM}$ ) on cardiac function, substrate utilization, and myocardial high energy phosphate levels was studied in 10 mongrel dogs. After $\mathbf{4 5}$ min of hypoglycemia, mean aortic pressure, total peripheral resistance, and myocardial oxygen consumption had increased significantly, but other hemodynamic parameters and regional myocardial function had not changed. Additional infusion of 3-hydroxybutyrate did not affect hemodynamic variables significantly. During both metabolic interventions in vivo phosphorus-31 nuclear magnetic resonance spectroscopy showed stable levels of myocardial phosphocreatinine, ATP, as well as the phosphocreatinine/ ATP (3.0-3.2) ratio. Biochemical measurements revealed that hyperketonemia led to significant alterations in arterial concentrations and arteriocoronary venous differences of selected citric acid cycle intermediates, thus confirming previous reports which suggested a blockade of the 2oxoglutarate-dehydrogenase reaction induced by ketone body oxidation. However, despite this blockade, the energy supply to the heart was not impaired as shown by normal nuclear magnetic resonance spectroscopy and cardiac performance. It is speculated, that the blockade might be due to an enhanced NADH/NAD ratio. (Pediatr Res 26:536542,1989 )
\end{abstract}

\section{Abbreviations}

a-cv, arteriocoronary venous

CBF, coronary blood now

HG, hypoglycemia

HK, hyperketonemia

ID, internal diameter

MAP, mean aortic pressure

MPAP, mean pulmonary arterial pressure

MSE, myocardial substrate extraction

$\mathrm{MVO}_{2}$, myocardial oxygen consumption

Received May 23, 1988; accepted July 17, 1989

Correspondence and reprints Kyung J. Chung, M.D., Department of Pediatrics, T-008-A, University of California at San Diego, School of Medicine, La Jolla, CA 92093.

Supported in part by the San Diego Radiology Research and Education Foundation. J. B. was a recipient of a scholarship from the Rotary International Foundation.

' Present address Division of Pediatric Cardiology, University of Tuebingen, Ruemelinstr. 21, 7400 Tuebingen, FRG.
NMR, nuclear magnetic resonance

OD, outer diameter

OER, oxygen extraction ratio

$\mathrm{PCr}$, phosphocreatinine

PVR, pulmonary yascular resistence

SV, stroke volume

TPR, total peripheral resistence

$\%$ S, systolic segmental shortening

An association of $\mathrm{HG}$ and acute heart failure or cardiac enlargement has been reported in newborns, infants, children, and adults $(1-8)$. The reason for this association is still obscure. Benzing et al. (3) have suggested that heart failure leads to $\mathrm{HG}$ because of a decreased dietary intake or malabsorption. Reid et al. (1) concluded that heart failure is induced by insufficient energy supply during HG (1). A decreased glucose availability should be compensated for by alternate energy substrates such as free fatty acids and ketone bodies. However, ketone bodies seem not to sustain full cardiac work-load $(9,10)$ because their oxidation is restricted within the citric acid cycle. Therefore, the combination of $\mathrm{HG}$ and a high concentration of ketone bodies which occurs in premature and dysmature infants $(11,12)$ may impair cardiac energy supply and performance, thereby leading to acute heart failure or cardiac enlargement (1-3). This hypothesis was tested by measuring global and regional myocardial function as well as myocardial high energy phosphates in dogs during acute $\mathrm{HG}$ and $\mathrm{HG}$ in the presence of hyperketonemia.

\section{MATERIALS AND METHODS}

Animal preparation. Experiments were performed in 10 mongrel dogs weighing $8-14 \mathrm{~kg}$. After fasting for approximately $4 \mathrm{~h}$, dogs were tranquilized with acepromazine maleate $(1.5 \mathrm{mg} / \mathrm{kg})$ $30 \mathrm{~min}$ before anesthesia with sodium pentobarbital (initial dose was $30 \mathrm{mg} / \mathrm{kg}$; additional doses of $5 \mathrm{mg} / \mathrm{kg}$ were administered when necessary). Mechanical ventilation was adjusted to maintain normal blood gases and acid base balance, and oxygen was supplemented when necessary. Body temperature was maintained by covering and external warming at $36-38^{\circ} \mathrm{C}$. ECG was monitored continously. Polyethylene catheters $(1.67 \mathrm{~mm} \mathrm{ID;}$ $2.41 \mathrm{~mm}$ OD) were inserted into the right femoral artery and vein. The arterial catheter was placed in the thoracic aorta for blood sampling and measurement of aortic pressure. The venous catheter was used for infusing drugs and fluids with a constant total infusion rate of $8-10 \mathrm{~mL} / \mathrm{kg} / \mathrm{h}$. To prevent interference with free fatty acid and carbohydrate metabolism, the use of 
heparin and solutions supplemented with lactate or glucose was strictly avoided.

A Swan-Ganz thermodilution catheter (Edwards Laboratories, Irvine, $\mathrm{CA}$ ) was advanced into the pulmonary artery via the left femoral vein for measurement of cardiac output, pulmonary artery pressure, and right atrial pressure. Cardiac output was determined in duplicate (Cardiac Output Computer, Edwards Laboratories, Irvine, CA) and the mean of both measurements was taken. A midline sternotomy was performed and another polyethylene catheter was inserted into the coronary sinus via the right atrium for blood sampling. The correct position of the coronary sinus catheter was confirmed after completion of the experimental protocol. A silicone rubber catheter $(1.27 \mathrm{~mm}$ ID; $2.28 \mathrm{~mm}$ OD) was placed directly into the left atrium for measurement of left atrial pressure. The pericardium was then sutured to the chest wall to cradle the heart. Regional myocardial function was assessed with a sonomicrometer (Triton Technologies, San Diego, CA) by measuring myocardial segmental length. A pair of ultrasonic crystals ( $5 \mathrm{mmHz}$ ) was implanted into each dog according to standard techniques (13) in the anterior wall of the left ventricle near the interventricular septum.

In five dogs subject to phosphorus-31 NMR spectroscopy, the Swan-Ganz thermodilution catheter was omitted to avoid disturbance of the homogeneity of the magnetic field. Moreover, a $2.5-\mathrm{cm}$ diameter double-turn circular radio frequency surface coil was sutured to the anterior wall of the left ventricle encircling the two ultrasonic crystals. These piezoelectric crystals and attached wires did not affect phosphorus-31 spectroscopy when disconnected from the sonomicrometer. Therefore, measurements of regional myocardial function and phosphorus-31 spectra were made alternately. Otherwise the preparation was the same as described above. After all preparations the chest was closed with a suture.

Biochemical studies. Arterial and coronary sinus blood samples were drawn simultaneously with heparinized syringes for measurement of oxygen saturation, blood gases, $\mathrm{Hb}$, and metabolic substrates. For determination of lactate, pyruvate, ketone bodies (i.e. 3-hydroxybutyrate and acetoacetate) and citric acid cycle intermediates, whole blood was immediately centrifuged. The plasma was then extracted with ice-cold, $0.4 \mathrm{M}$ perchloric acid, and the supernatant was stored at $-70^{\circ} \mathrm{C}$ until it was assayed on the next day with a recently described HPLC method (14). Glucose determinations were performed by the glucose-oxidase method with an automatic analyzer (ASTRA, Beckman, Fullerton, CA). Serum samples for free fatty acid analysis were stored at $-10^{\circ} \mathrm{C}$ and assayed within $10 \mathrm{~d}$ by the colorimetric method of Duncombe (15).

NMR studies. For in vivo phosphorus-31 NMR measurements, a 23.5-cm horizontal bore, 2.0 Tesla superconducting magnet (Oxford Instruments, Oxford, UK) and a General Electric (Milwaukee, WI) computer were used. The phosphorus-31 resonance frequency of this system was $34.5 \mathrm{mHz}$. After implantation of the surface coil the dog was wrapped into a plastic bag and positioned with its heart in the center of the magnet. The magnetic homogeneity in the region of the surface coil was optimized by shimming on the proton signal. NMR data were collected over $17 \mathrm{~min}$, using a 4-s pulse delay and a $35-\mathrm{ms}$ pulse width. The spectra obtained during hemodynamic and metabolic steady states were analysed by measuring the peak ht from baseline while maintaining all parameters constant throughout the experiment. Peak intensities were used, because, consistent with signal to noise ratio and spectral overlap, the intensities were found to have better reproducibility than did resonance areas (16).

Experimental protocol. After completion of surgery, the dog was allowed to stabilize for $20 \mathrm{~min}$, then the first control measurement (control) was performed including all hemodynamic parameters and simultaneous blood sampling from the aorta and the coronary sinus. Then, $1 \mathrm{mg} / \mathrm{kg}$ propranolol was injected to prevent direct metabolic effects of changes in sympathetic tone, mediated via $\beta$-receptors. After $15 \mathrm{~min}$, a second measurement (after propranolol) was made. Thereafter, $\mathrm{HG}$ was induced by an injection of $20 \mathrm{U} / \mathrm{kg}$ regular insulin; and a continous infusion of $1 \mathrm{U} / \mathrm{kg} / \mathrm{h}$ insulin and $1.2 \mathrm{mmol} / \mathrm{kg} / \mathrm{h}$ potassium dissolved in $0.9 \%$ saline was started. With this supplementation of potassium the serum concentration was kept constant at a normal level of $3.7 \pm 0.3 \mathrm{mM}$. Blood glucose concentrations were monitored every $10-15 \mathrm{~min}$ after insulin injection with chemistrip-bG (Boehringer Mannheim Diagnostics, FRG) until HG was accomplished, defined as glucose levels of $30 \mathrm{mg} / \mathrm{dL}$. Forty-five min after the first glucose determination had shown a value of 30 $\mathrm{mg} / \mathrm{dL}$, the third measurement was made. A low level of $\mathrm{HK}$ (low HK) was then induced by infusing sodium DL-3-hydroxybutyrate (Sigma Chemical Co., St. Louis, MO) at a rate of $6 \mathrm{mg} /$ $\mathrm{kg} / \mathrm{min}$ for $10 \mathrm{~min}$ and $3 \mathrm{mg} / \mathrm{kg} / \mathrm{min}$ for additional $35 \mathrm{~min}$. Sodium 3-hydroxybutyrate was dissolved in purified water and adjusted to a $\mathrm{pH}$ of 7.40 by adding appropriate amounts of $1 \mathrm{~N}$ hydrochloric acid. Fifteen min after starting the infusion of 3 hydroxybutyrate, a constant plasma concentration of ketone bodies was established and $30 \mathrm{~min}$ later the fourth measurement ( $\mathrm{HG}+$ low $\mathrm{HK}$ ) was performed. The infusion rate of 3 -hydroxybutyrate was then increased to $6 \mathrm{mg} / \mathrm{kg} / \mathrm{min}$ to obtain a higher level of $\mathrm{HK}$ (high $\mathrm{HK}$ ). This was accomplished after another 30 min and a measurement ( $\mathrm{HG}+$ high $\mathrm{HK}$ ) was made $15 \mathrm{~min}$ later. At the last step of our protocol the dog was allowed to recover from $H G$ and $H K$ by ending both infusions and starting a $20 \%$ dextrose infusion with a rate of 1 to $1.5 \mathrm{~mL} / \mathrm{min}$. After 60 min the last measurement (recovery) was performed. NMR data acquisition were started after the hemodynamic and metabolic measurements had been completed leading to a 17 min prolongation of each step of the protocol.

Data acquisition and calculations. Data were recorded on a 8 channel recorder (Gould, Cleveland, $\mathrm{OH}$ ) or on a Mingograf 91 (Elma-Schoenander, Stockholm, Sweden), and digitized manually. MAP and MPAP were either electronically derived from the pressure signal or calculated. SV, TPR, and PVR were calculated according to standard equations. \%S was defined as the difference between end-systolic and end-diastolic segmental length, expressed as percentage of the end-diastolic segmental length. MVO was calculated from the formula MVO $(\mathrm{mL} \mathrm{O} /$ $100 \mathrm{~g} \cdot \min )=0.00072 \times$ systolic aortic pressure $\times$ heart rate + $1.43(17)$. Blood oxygen content was calculated as $\mathrm{O}(\mathrm{mL} / \mathrm{dL})=$ saturation $\times \mathrm{Hb} \times 1.36$; and carbon dioxide content $(\mathrm{mL} / \mathrm{dL})$ was determined from the pco using the Hendersson-Hasselbalch equation $(18,19)$ and a factor converting plasma CO content into blood CO content (20). CBF was estimated (est. CBF) from the formula est. CBF $(\mathrm{mL} / 100 \mathrm{~g} \cdot \mathrm{min})=\mathrm{MVO} / \mathrm{a}-\mathrm{c} \mathrm{v}$ oxygen difference. The myocardial respiratory quotient, i.e. the a-cv $\mathrm{CO}$ difference divided by the a-cv $O$ difference, was also calculated. MSE was defined as the a-cv substrate difference divided by the corresponding arterial concentration. The percent contribution of different substrates to the aerobic metabolism of the myocardium was estimated by the OER, i.e. oxygen equivalents of a substrate divided by the a-cv $\mathrm{O}$ difference $(21)$. With this ratio, the most important myocardial fuels at each experimental condition can be readily recognized. The following oxygen equivalents were used: $3 \mathrm{~mol} \mathrm{O} / \mathrm{mol}$ lactate, $2.5 \mathrm{~mol} \mathrm{O} / \mathrm{mol}$ pyruvate, $4.5 \mathrm{~mol} \mathrm{O} / \mathrm{mol}$ ketone bodies, $6 \mathrm{~mol} \mathrm{O} / \mathrm{mol}$ glucose, and $23 \mathrm{~mol}$ $O / \mathrm{mol}$ free fatty acids. Mitochondrial respiration was assessed by PCr/ATP (22-24).

Statistics. Statistical analysis was performed by using the BMDP statistical software, installed on a Siemens 7.580 mainframe. Mean values are given with the SEM. Differences between arterial and coronary venous substrate concentrations at each step of the protocol were statistically verified by paired $t$ tests. A regression analysis was performed on arterial concentrations of selected citric acid cycle intermediates versus ketone body concentration. Variations between the six steps of the experimental protocol were statistically tested by an a priori analysis, the method of orthogonal contrasts (25). In advance of collecting the 